\title{
"COVID-19: diagnosis, management and prognosis": a new topical collection of Internal and Emergency Medicine
}

\author{
Riccardo Polosa ${ }^{1,2}$ (1) Michele Spinicci ${ }^{3,4} \cdot$ Domenico Prisco ${ }^{3,5}$
}

Received: 14 July 2020 / Accepted: 23 July 2020 / Published online: 30 July 2020

(c) Società Italiana di Medicina Interna (SIMI) 2020

At the dawn of 2020, the planet woke up with an unexpected shocking public health threat. A novel zoonotic coronavirus, later named severe acute respiratory syndrome 2 (SARSCoV-2), genetically close to SARS-CoV responsible for the outbreak in 2003, emerged as causative agent of a multifaceted syndrome, known as coronavirus disease 2019 (COVID-19) [1].

The outbreak rapidly spread from the Wuhan City, Hubei Province, China, where the first cases of SARS-CoV-2 infection occurred in early December 2019, toward western countries, resulting in an unprecedented challenge for the health systems worldwide. On 11th March 2020, the World Health Organization declared the outbreak of SARS-CoV-2 a pandemic. As of the 8 th of July 2020 globally more than 11 million cases of SARS-CoV-2 infections had been confirmed, and 545,398 COVID-19 related fatalities had occurred globally [2].

The body of knowledge about biologic SARS-CoV-2 and clinical features of COVID-19 has been increasing exponentially. Nonetheless, conflicting opinions and confusing issues still dominate the scientific debate. The topical collection of Internal and Emergency Medicine, titled "COVID-19: diagnosis, management and prognosis" was launched to better

The article belongs to COVID- 19 .

Riccardo Polosa

polosa@unict.it

1 Dipartimento Di Medicina Clinica E Sperimentale (MEDCLIN), University of Catania, Catania, Italy

2 Center of Excellence for the Acceleration of HArm Reduction (CoEHAR), University of Catania, Catania, Italy

3 Dipartimento Di Medicina Sperimentale E Clinica (DMSC), Università Di Firenze, Florence, Italy

4 SOD Malattie Infettive E Tropicali, Azienda Ospedaliero-Universitaria Careggi, Florence, Italy

5 SOD Medicina Interna Interdisciplinare, Azienda Ospedaliero-Universitaria Careggi, Florence, Italy inform researchers, academics, physicians and healthcare professionals about the evolving understanding of the topic.

Ethical issues are at the forefront of the debate, as it deserves for an event of such magnitude, to whom healthcare workers (HCWs) paid a heavy price. In their fascinating letter, Lippi et al. offered a historical perspective of doctors' role in the past epidemics, stuck between professional duties, based on the Hippocratic Oath, and the need to protect themselves, through adequate measures and equipment [3]. More to the point, Arora et al. identified ethics key issues in the COVID-19 era, such as the long-term implications of nonurgent care cancelled, how to make priority decisions on patients' treatment escalation and how clinicians should act when adequate personal protective equipment (PPE) is not available [4].

Indeed, as highlighted by Russo et al., the pandemic storm has been not only a clinical challenge but also an organizational crisis for our health systems [5]. Shortage of PPE for frontline HCWs was one of the more worrisome aspects of the early pandemic. Tsilingiris et al. went into the debate on the appropriate use of medical masks and respirators for HCWs and revised available evidence on the topic [6]. The theme of COVID-19 as diversion and deterrent from the care of patients with chronic conditions, causing possible negative consequences in the near future, returned in the point of view by Viganò et al.: in their Emergency Department, the admissions dropped in March and April 2020 by $53 \%$ and $63 \%$, respectively, with respect to the period December 2019-February 2020 [7]. Two nice papers rose concern on how pandemic can represent an additional complication for the management of cardiac emergencies, both inside and outside the hospitals $[8,9]$. The experience of the first 5 weeks of COVID-19 epidemic in a referral centre in the epicentre of the Italian epidemic represented an effective strategy of Emergency Medicine network, able to manage an increased amount of calls and requests, by integrating "outof-hospital" and "hospital" efforts [10]. Within the hospitals, physicians from disparate specialities were called to change 
their habits, when their facilities and wards were converted to COVID-19 areas, in response to an overwhelming flow of affected patients. At best, it was an occasion to put in place an unexpected teamwork cooperation and to share different skills and experiences [11].

The correct identification of COVID-19 cases remains the cornerstone for the control of the epidemic, allowing to confine infected patients and prevent SARS-CoV-2 spread. At the hospital level, an effective triage strategy is of paramount importance to avoid nosocomial outbreaks amongst HCWs and patients with other diseases than COVID-19. Poggiali et al. described their flowchart, applied in the heart of the Italian epidemic storm [12]. Polymerase chain reaction (PCR) testing on respiratory samples, i.e., nasopharyngeal swab, sputum or bronchoalveolar lavage, is the gold standard for a confirmed diagnosis of SARS-CoV-2 infection. However, physicians soon learned that in some cases, a typical clinical and radiological picture should drive the diagnosis, regardless of a negative microbiological result. As an example, in the case report by Song et al. a patient with fever, respiratory symptoms and evocative mixed ground-glass opacity at the computed tomography (CT), had negative PCR on seven consecutive sputum sample, before achieving the diagnosis of SARS-CoV-2 infection on the eighth sample [13].

Typical and atypical clinical features of COVID-19 patients, as well as risk factors with possible impact on COVID-19 outcome, are outlined in several papers of this Topical Collection. Bertolino et al. tried to summarize the evidences on key clinical features to differentiate COVID-19 case from upper respiratory and/or influenza-like illnesses of other aetiology, providing a practical guide for internists [14]. According to data collected by Lapostolle et al. among 1487 outpatients diagnosed with COVID-19 in the Greater Paris region, dry cough and fever were the most frequent symptoms reported (more than $90 \%$ of cases), in association with general symptoms, such as body aches/myalgia, headache, and asthenia, and a considerable quote of anosmia and ageusia [15]. Nevertheless, the clinical presentation can be sometimes atypical, respiratory symptoms can be less pronounced, and other sites targeted, such as the digestive tract, the cardiovascular system and central nervous system. For instance, a number of neurologic manifestation, from unspecific symptoms such as headache, altered mental status, and anosmia, up to cerebrovascular accidents and Guillain-Barré syndrome have occurred in many patients with Covid-19 $[16,17]$. Morjaria et al. described two cases of unusual neurological complications, i.e. bilateral lower limbs weakness, possibly related to cerebrovascular accidents, in critically ill patients with prolonged hospital stays [18]. Great interest exists on whether patients presenting with atypical symptoms are at increased risk to evolve toward severe forms of COVID-19. Henry et al. revised the available literature about common gastrointestinal symptoms and found that abdominal pain, nausea and vomiting, but not diarrhoea, were significantly associated with an increased odds of severe COVID-19 [19]. Moreover, myocardial injury was often observed in SARS-CoV-2 patients, especially those with severe to critical disease, in need of intensive care treatment. Preliminary data by Violi et al. on a limited sample of patients suggested that patients who show troponin elevation may be at higher risk of mortality [20]. As for other predictors of outcome in COVID-19 patients, Sciacqua et al. explored the potential causative factors of poor outcome in elderly people, such as immunosenescence, reduced resilience and the coexistence of multiple comorbidities, and focused on impaired nutritional status and sarcopenia, commonly due to inadequate food and calorie intake and an unmet increased protein demand [21]. Pantanetti et al. reviewed the pathophysiologic basis related to an excess of disease severity in diabetic patients and suggested a possible use of dipeptidyl peptidase-4 inhibitors as immunomodulatory drugs in COVID-19 [22]. Surprisingly, a systematic review by Farsalinos et al. found that smoking had a protective effect against COVID-19, based on an unexpected low prevalence of current smokers among Chinese patients hospitalized with COVID-19 [23]. Despite confidence issues on the accuracy about data collection methods for reporting smoking status, similar findings have been reported from other countries, allowing to speculate on pharmaceutical nicotine as a potential treatment option in COVID-19 [24].

To date, there is no established treatment for COVID19. In recent days, remdesivir was the first antiviral drug approved by U.S. Food and Drug Administration (FDA) and European Medicine Agency (EMA) for the treatment of hospitalized adults with evidence of SARS-CoV-2 lower respiratory tract involvement, in virtue of the positive results obtained in a double-blind, randomized, placebo-controlled trial [25]. So far, remdesivir has been available only for compassionate use or within the randomized clinical trial and therapeutic strategies have largely relied on off-label use of old drugs, such as antimalarial drugs chloroquine (CQ) and hydroxychloroquine (HCQ) and antiretroviral lopinavir/ ritonavir and darunavir/cobicistat [26]. Unfortunately, conclusive data about the real impact of these compounds on the outcome on Covid-19 patients are still lacking, albeit a constant flow of published data from clinical experiences, mostly biased by methodological flaws. Fanin et al. analysed ins and outs of a well-known open-label non-randomized clinical trial by Gautret et al. on the efficacy of the combination use of hydroxychloroquine and azithromycin, which had great resonance and influenced treatment strategies worldwide, despite important limitations [27, 28]. Depfenhart et al. revised pharmacodynamics basis of antiviral drugs available so far, and suggested bromhexine, an FDA-approved ingredient in mucolytic cough suppressants, as a potential new option, due to its TMPRSS2 inhibitor activity [29]. 
However, overall management of Covid-19 patients exceeds the administration of antiviral drugs. Immunemodulant drugs and anti-thrombotic prophylaxis are pillars of the treatment of severe forms. Both arterial and venous thrombotic events frequently complicated the course of patients with COVID-19, especially in those critically ill, leading to the definition of Coronavirus-associated coagulopathy (CAC) by the International Society on Thrombosis and Haemostasis [30, 31]. An interesting Spanish case series of non-ICU hospitalized COVID-19 patients diagnosed with pulmonary embolism, without evidence of concomitant deep-vein thrombosis of the lower limbs, suggested a predominance of small-vessel thrombosis secondary to inflammatory and immune responses [32]. Understanding mechanism underlying CAC is crucial to put in place appropriate therapeutic measures: observed hemostatic alternations-increased D-dimer, normal or slightly deranged prothrombin time and within range platelets count-differ from those usually observed during the disseminated intravascular coagulopathy. Bazzan et al. found reduced levels of ADAMTS-13 and higher levels of von Willebrand factor in a cohort of COVID-19 patients, in comparison with healthy controls, and also in fatal COVID-19 cases, when compared to patients with the non-fatal outcome. These alterations, similar to those observed in patients with thrombotic thrombocytopenic purpura, may lean more to a thrombotic microangiopathy origin of CAC, rather than a consumption coagulopathy [33]. Moreover, the infusion of hyperimmune plasma from donors recovered from SARS-CoV-2 infection emerged as an attractive option. Perotti et al. presented the COVID-19 PLASMA trial, the first proof-of-concept interventional trial using hyperimmune plasma with a high titre of specific neutralizing antibodies for treating critical patients with COVID-19 [34]. Supportive care includes supplemental oxygen and ventilatory management of patients with respiratory distress. Privitera et al. proposed a flowchart for non-invasive ventilation support in COVID-19 patients, as a first approach in the Emergency Department [35].

Management of chronic therapies was also challenging. The role of ACE- 2 receptor in SARS-CoV-2 cell entry process produced a lively debate on the potential impact of angiotensin-converting enzyme inhibitors (ACE-Is) and angiotensin II receptor blockers (ARBs), extensively used for the treatment of hypertension and other cardiovascular diseases, on the natural history of SARS-Cov-2 infection. Two papers of the Topical Collection explored the potential implication of ACE-Is and ARBs use in Covid-19 patients [36, 37]. Finally, Testa et al. addressed the management of Covid-19 patients on oral anticoagulant drugs, and the high risk of over/under treatment due to the multiple pharmacological interactions, and the possible necessity of mechanical ventilation, and suggested replacing oral anticoagulant therapies with parenteral low-molecular-weight heparin or unfractionated heparin [38].
On the other hand, a paper by Poli et al. provided some advice aimed at improving the outpatient management of people on anticoagulant treatment during COVID-19 pandemic, with particular regard to the lockdown and reopening periods [39].

In the authors' view, this new Topical Collection will contribute significantly to stimulate the scientific debate and to advance the current body of knowledge of the medical community about the pandemic and its ethical, organizational, and clinical challenges. Given the importance of the topic to the active role that internists and general physicians play in assisting the many patients directly or indirectly affected by COVID-19, Internal and Emergency Medicine remains committed to further expanding the current knowledge base and advancing the scientific debate about the impact of SARSCoV-2 on human health worldwide.

\section{Compliance with ethical standards}

Conflict of interest RP is full-time employee at the University of Catania, Italy. In relation to the topic of communicable disease, RP has received research funding from Alfa-Wassermann, manufacturer of broad-spectrum antibiotics. RP is also the founder of the Center of Excellence for the acceleration of Harm Reduction at the University of Catania (CoEHAR), which has received a grant from Foundation for a Smoke-Free World to develop and carry out 8 research projects and scientific advisor for LIAF, Lega Italiana Anti Fumo (Italian acronym for Italian Anti-Smoking League). MS and DP declare no conflicts of interest.

Statement of human and animal rights This article does not contain any studies with human or animal subjects performed by any of the authors.

Informed consent Informed consent was not required for this type of study.

\section{References}

1. Zhu N, Zhang D, Wang W et al (2020) A novel coronavirus from patients with pneumonia in China, 2019. N Engl J Med 382(8):727-733. https://doi.org/10.1056/NEJMoa2001017

2. World Health Organization (2020) Coronavirus disease (COVID2019) situation reports. https://www.who.int/emergencies/disea ses/novel-coronavirus-2019/situation-reports/ Last access: 08th July 2020

3. Lippi D, Bianucci R, Donell S (2020) Role of doctors in epidemics: historical perspectives and implications for COVID-19. Intern Emerg Med. https://doi.org/10.1007/s11739-020-02351-x

4. Arora A, Arora A (2020) Ethics in the age of COVID-19. Intern Emerg Med. https://doi.org/10.1007/s11739-020-02368-2

5. Russo E, Brogi E, Gamberini E et al (2020) COVID-19: a clinical and organizational crisis. Intern Emerg Med. https://doi. org/10.1007/s11739-020-02410-3

6. Tsilingiris D, Papatheodoridi M, Kapelios CJ (2020) Providing evidence on the ongoing health care workers' mask debate. Intern Emerg Med. https://doi.org/10.1007/s11739-020-02382-4 
7. Mauro V, Lorenzo M, Paolo C et al (2020) Treat all COVID 19-positive patients, but do not forget those negative with chronic diseases. Intern Emerg Med. https://doi.org/10.1007/s11739-020-02395-z

8. Pranata R, Lim MA, Yonas E et al (2020) Out-of-hospital cardiac arrest prognosis during the COVID-19 pandemic. Intern Emerg Med. https://doi.org/10.1007/s11739-020-02428-7

9. Sgura FA, Arrotti S, Cappello CG et al (2020) Complicated myocardial infarction in a 99-year-old lady in the era of COVID-19 pandemic: from the need to rule out coronavirus infection to emergency percutaneous coronary angioplasty. Intern Emerg Med. https://doi.org/10.1007/s11739-020-02362-8

10. Perlini S, Canevari F, Cortesi S et al (2020) Emergency department and out-of-hospital emergency system (112-AREU 118) integrated response to coronavirus disease 2019 in a Northern Italy centre. Intern Emerg Med. https://doi.org/10.1007/s1173 9-020-02390-4

11. Cogliati C, Ceriani E, Brambilla AM (2020) When internal and emergency medicine speak to each other: organization in the time of COVID. Intern Emerg Med. https://doi.org/10.1007/s1173 9-020-02380-6

12. Erika P, Andrea V, Cillis MG et al (2020) Triage decision-making at the time of COVID-19 infection: the Piacenza strategy. Intern Emerg Med. https://doi.org/10.1007/s11739-020-02350-y

13. Song C, Yang D, Lu Y (2020) A COVID-19 patient with seven consecutive false-negative rRT-PCR results from sputum specimens. Intern Emerg Med. https://doi.org/10.1007/s11739-02002423-y

14. Bertolino L, Vitrone M, Durante-Mangoni E (2020) Does this patient have COVID-19? A practical guide for the internist. Intern Emerg Med. https://doi.org/10.1007/s11739-020-02377-1

15. Lapostolle F, Schneider E, Vianu I et al (2020) Clinical features of 1487 COVID-19 patients with outpatient management in the Greater Paris: the COVID-call study. Intern Emerg Med. https:// doi.org/10.1007/s11739-020-02379-z

16. Toscano G, Palmerini F, Ravaglia S et al (2020) GuillainBarré syndrome associated with SARS-CoV-2. N Engl J Med 382(26):2574-2576. https://doi.org/10.1056/NEJMc2009191

17. Mao L, Jin H, Wang $M$ et al (2020) Neurologic manifestations of hospitalized patients with coronavirus disease 2019 in Wuhan China. JAMA Neurol 77(6):1-9. https://doi.org/10.1001/jaman eurol.2020.1127 (published online ahead of print, 2020 Apr 10)

18. Morjaria JB, Omar F, Polosa R et al (2020) Bilateral lower limb weakness: a cerebrovascular consequence of covid-19 or a complication associated with it? Intern Emerg Med. https://doi. org/10.1007/s11739-020-02418-9

19. Henry BM, de Oliveira MHS, Benoit J et al (2020) Gastrointestinal symptoms associated with severity of coronavirus disease 2019 (COVID-19): a pooled analysis. Intern Emerg Med. https:// doi.org/10.1007/s11739-020-02329-9

20. Violi F, Pastori D, Pignatelli P et al (2020) SARS-CoV-2 and myocardial injury: a role for Nox2? Intern Emerg Med. https:// doi.org/10.1007/s11739-020-02348-6

21. Sciacqua A, Pujia R, Arturi F et al (2020) COVID-19 and elderly: beyond the respiratory drama. Intern Emerg Med. https://doi. org/10.1007/s11739-020-02424-x

22. Pantanetti P, Cangelosi G, Ambrosio G (2020) Potential role of incretins in diabetes and COVID-19 infection: a hypothesis worth exploring. Intern Emerg Med. https://doi.org/10.1007/s1173 9-020-02389-x

23. Farsalinos K, Barbouni A, Niaura R (2020) Systematic review of the prevalence of current smoking among hospitalized COVID-19 patients in China: could nicotine be a therapeutic option? Intern Emerg Med. https://doi.org/10.1007/s11739-020-02355-7

24. Polosa R, Caci G (2020) COVID-19: counter-intuitive data on smoking prevalence and therapeutic implications for nicotine. Intern Emerg Med. https://doi.org/10.1007/s11739-020-02361-9
25. Beigel JH, Tomashek KM, Dodd LE et al (2020) Remdesivir for the treatment of Covid-19-preliminary report. N Engl J Med. https://doi.org/10.1056/NEJMoa2007764 (published online ahead of print, 2020 May 22)

26. Galluccio F, Ergonenc T, Garcia Martos A et al (2020) Treatment algorithm for COVID-19: a multidisciplinary point of view. Clin Rheumatol 39(7):2077-2084. https://doi.org/10.1007/s1006 7-020-05179-0

27. Fanin A, Calegari J, Beverina A et al (2020) Hydroxychloroquine and azithromycin as a treatment of COVID-19. Intern Emerg Med. https://doi.org/10.1007/s11739-020-02388-y

28. Gautret P, Lagier JC, Parola P et al (2020) Clinical and microbiological effect of a combination of hydroxychloroquine and azithromycin in 80 COVID-19 patients with at least a six-day follow up: a pilot observational study. Travel Med Infect Dis 34:101663. https://doi.org/10.1016/j.tmaid.2020.101663

29. Depfenhart M, de Villiers D, Lemperle G et al (2020) Potential new treatment strategies for COVID-19: is there a role for bromhexine as add-on therapy? Intern Emerg Med. https://doi. org/10.1007/s11739-020-02383-3

30. Landi A, De Servi S (2020) The burden of thrombotic complications in critically ill patients with COVID-19: charting the uncharted. Intern Emerg Med. https://doi.org/10.1007/s1173 9-020-02393-1

31. Tachil J, Tang N, Gando S et al (2020) ISTH interim guidance on recognition and management of coagulopathy of Covid 19. JTH 18:1023-1026

32. Dubois-Silva Á, Barbagelata-López C, Mena Á et al (2020) Pulmonary embolism and screening for concomitant proximal deep vein thrombosis in noncritically ill hospitalized patients with coronavirus disease 2019. Intern Emerg Med. https://doi.org/10.1007/ s11739-020-02416-x

33. Bazzan M, Montaruli B, Sciascia S et al (2020) Low ADAMTS 13 plasma levels are predictors of mortality in COVID-19 patients. Intern Emerg Med. https://doi.org/10.1007/s11739-020-02394-0

34. Perotti C, Del Fante C, Baldanti F et al (2020) Plasma from donors recovered from the new Coronavirus 2019 as therapy for critical patients with COVID-19 (COVID-19 plasma study): a multicentre study protocol. Intern Emerg Med. https://doi.org/10.1007/s1173 9-020-02384-2

35. Privitera D, Angaroni L, Capsoni N et al (2020) Flowchart for non-invasive ventilation support in COVID-19 patients from a northern Italy emergency department. Intern Emerg Med. https ://doi.org/10.1007/s11739-020-02370-8

36. Sarzani R, Giulietti F, Di Pentima C et al (2020) Antagonizing the renin-angiotensin-aldosterone system in the era of COVID-19. Intern Emerg Med. https://doi.org/10.1007/s11739-020-02365-5

37. Albini A, Di Guardo G, Noonan DM et al (2020) The SARSCoV-2 receptor, ACE-2, is expressed on many different cell types: implications for ACE-inhibitor- and angiotensin II receptor blocker-based cardiovascular therapies. Intern Emerg Med. https ://doi.org/10.1007/s11739-020-02364-6

38. Testa S, Paoletti O, Giorgi-Pierfranceschi M et al (2020) Switch from oral anticoagulants to parenteral heparin in SARS-CoV-2 hospitalized patients. Intern Emerg Med. https://doi.org/10.1007/ s11739-020-02331-1

39. Poli D, Tosetto A, Palareti G et al (2020) Managing anticoagulation in the COVID-19 era between lockdown and reopening phases. Intern Emerg Med. https://doi.org/10.1007/s11739-02002391-3

Publisher's Note Springer Nature remains neutral with regard to jurisdictional claims in published maps and institutional affiliations. 